\title{
Particularités physiologiques de la souche CNRZ 125 de Lactococcus lactis subsp lactis biovar diacetylactis
}

\author{
C Foucaud *, M Rousseau, S Furlan, D Hemme \\ INRA, Station de recherches laitières, 78350 Jouy-en-Josas, France
}

(Reçu le 14 février 1990; accepté le 24 août 1990)

\begin{abstract}
Résumé - Lactococcus lactis subsp lactis biovar diacetylactis CNRZ 125 est une souche qui perd facilement et uniquement le plasmide codant pour le métabolisme du lactose, en conditions de privation nutritionnelle. Cette étude décrit d'autres particularités de sa physiologie, comparativement à la souche Lc lactis subsp lactis CNRZ 141 (ML3). La perte de viabilité, les changements morphologiques, la dégradation des protéines et des acides aminés intracellulaires, la perte d'acides aminés et d'activité de la lactate déshydrogénase sont plus faibles chez la souche CNRZ 125 que chez la souche CNRZ 141. Les chaînes de la souche CNRZ 125 sont plus longues et témoignent d'une activité autolytique faible que pourrait expliquer la présence d'une couche périphérique extérieure à la paroi. Les cellules des souches CNRZ 125 et CNRZ 141 prélevées au cours de la phase exponentielle de croissance perdent plus rapidement leur viabilité que celles prélevées plus tardivement. Cette perte de viabilité ne peut être associée à celle d'une fonction ou d'un constituant cellulaire particulier et elle est plus importante pour les cellules cultivées en milieu glucosé plutôt que lactosé ou galactosé.
\end{abstract}

Lactococcus lactis biovar diacetylactis / privation nutritionnelle / viabilité / dégradation de macromolécules / paroi

Summary - Particular physiological aspects of Lactococcus lactis subsp lactis biovar diacetylactis CNRZ 125. Lactococcus lactis subsp lactis biovar diacetylactis CNRZ 125 are a strain which easily loses its plasmid encoding for lactose metabolism during starvation. This study describes other particular aspects of its physiology which are then compared to that of Lc lactis subsp lactis CNRZ 141. Loss of viability, degree of morphological changes, protein and intracellular amino acids degradation and release of amino acids and lactate dehydrogenase activity are lower in strain CNRZ 125 than in strain CNRZ 141. The chains of Lc lactis CNRZ 125 are longer than those of CNRZ 141 and so reflect a weaker autolytic activity which could be explained by the presence of a aslime. The survival rate of exponential phase cells of both strains is lower than that of stationary phase cells. The loss of viability cannot be correlated to the release of a particular cellular constituent or function. Futhermore, glucose grown cells showed a longer survival rate than lactose or galactose grown cells.

Lactococcus lactis biovar diacetylactis / starvation / survival / macromolecule degradation / cell wall

\footnotetext{
* Correspondance et tirés à part
} 


\section{INTRODUCTION}

Lactococcus lactis, du fait de son large emploi dans le domaine de la technologie fromagère (Lenoir et $a l, 1985$ ) est l'espèce de bactéries lactiques la plus étudiée. Toutefois, à côté d'études génétiques de plus en plus développées, celles concernant le métabolisme sont le plus souvent relatives à des cellules qui se développent dans des conditions favorables à leur croissance.

Dans les produits laitiers, les bactéries lactiques mésophiles ne sont jamais dans des conditions optimales de croissance et se trouvent même très rapidement dans des conditions de survie. Le fromage est un milieu acide qui contient des concentrations croissantes de chlorure de sodium et qui séjourne, au cours de l'affinage, à des températures basses (Choisy et al, 1987). Les cellules subsistent ainsi dans des conditions qui ne sont plus favorables à leur multiplication, notamment dans des conditions de privation nutritionnelle avec le plus souvent absence de source glucidique (Choisy et al, 1987).

Peu de travaux ont porté sur la physiologie de Lc lactis en conditions de privation nutritionnelle. Après les études exhaustives de Thomas et Batt $(1968,1969)$ et de Thomas et al (1969) qui n'ont considéré que la seule souche Lc lactis ML3, les travaux dans ce domaine ont essentiellement porté sur le comportement autolytique de différentes souches de Lc lactis (Vegarud et al, 1983; Langsrud et al, 1987; Niskasaari, 1989). Qui plus est, peu de travaux concernent spécifiquement la variété diacetylactis de Lc lactis (Moustafa et Collins, 1968). Une étude récente a montré que la souche CNRZ 125 de la variété diacetylactis de Lc lactis perdait spécifiquement le plasmide codant pour le métabolisme du lactose et ceci, plus facilement en conditions de privation nutrition- nelle (Foucaud et al, 1990). La présente étude montre que la physiologie de cette souche, notamment dans des conditions de privation nutritionnelle, présente des différences importantes avec celle de Lc lactis CNRZ 141 (également ML3).

\section{MATÉRIEL ET MÉTHODES}

\section{Organismes et conditions de culture}

Lc lactis subsp lactis biovar diacetylactis CNRZ 125 et LC lactis subsp lactis CNRZ 141 (également ML3) provenaient de la collection INRA du Centre de recherches de Jouy-en-Josas (exCNRZ), France, et étaient conservées sur lait tournesolé à $-20^{\circ} \mathrm{C}$.

Les cultures bactériennes étaient réalisées en milieu Elliker (Biokar) ou M 17 préparé selon Terzaghi et Sandine (1975) et supplémenté en lactose $\left(5,10\right.$ ou $\left.50 \mathrm{~g}^{-1}\right)$, glucose ou galactose $\left(5 \mathrm{~g}^{\circ} \mathrm{l}^{-1}\right)$ ensemencé à $1 \%$ avec une préculture effectuée dans le même milieu pendant $10 \mathrm{~h}$ à $30^{\circ} \mathrm{C}$.

Le suivi de la croissance bactérienne s'effectuait par mesure du $\mathrm{pH}$ et de la densité optique à $650 \mathrm{~nm}\left(\mathrm{DO}_{650}\right.$ comprise entre 0,4 et 0,6$)$ de la culture ou d'une dilution de celle-ci, réalisée avec le milieu de culture non ensemencé.

\section{Conditions de privation nutritionnelle}

Les cellules étaient récoltées par centrifugation ( $10000 \mathrm{~g}, 20 \mathrm{~min}, 4^{\circ} \mathrm{C}$ ) après 3 ou $6 \mathrm{~h}$ de croissance (phase exponentielle) ou 12 ou $18 \mathrm{~h}$ (phase stationnaire). Elles étaient lavées 2 fois en tampon phosphate de potassium $50 \mathrm{mM} \mathrm{pH}$ 6 puis remises en suspension dans le même tampon. $\mathrm{La} \mathrm{DO}_{650}$ était ajustée à 3 soit $5,0 \times 10^{8}$ ufc $\cdot \mathrm{ml}^{-1}$ ou $1,2 \mathrm{mg}$ de poids sec $\mathrm{ml}^{-1}$ (souche CNRZ 125) et $11,8 \times 10^{8}$ ufc $\cdot \mathrm{ml}^{-1}$ ou $1,0 \mathrm{mg} \mathrm{de}$ poids sec $\cdot \mathrm{ml}^{-1}$ (souche CNRZ 141).

La suspension cellulaire était incubée à $30^{\circ} \mathrm{C}$ sans agitation et des fractions aliquotes étaient prélevées à différents moments par centrifugation et filtration pour analyse du surnageant et du culot bactérien, préalablement lavé avec de l'eau distillée. 


\section{Procédés d'analyse}

La viabilité était déterminée par étalement des dilutions appropriées de l'échantillon sur milieu M 17 ou Elliker gélosé et dénombrement des colonies bactériennes après incubation des boîtes à $30^{\circ} \mathrm{C}$ pendant $48 \mathrm{~h}$.

Le nombre de cellules par chaîne était estimé par comptage en microscopie photonique en contraste de phase (grossissement 1000x) des cellules de 300 chaînes colorées au bleu de méthylène $(0,6 \%$ dans l'éthanol absolu).

Les observations en microscopie électronique étaient réalisées sur des coupes fines des organismes. Les cultures des 2 souches, arrêtées au cours de la phase exponentielle de croissance $(3 \mathrm{~h})$, étaient transférées en tampon phosphate. Elles étaient ensuite récoltées par centrifugation à intervalles réguliers, lavées dans la même solution puis en tampon cacodylate de sodium $10 \mathrm{mM} \mathrm{pH} \mathrm{7,2.} \mathrm{Le} \mathrm{culot} \mathrm{cellulaire}$ (environ $10^{10}$ ufc) était enrobé avec de la gélose à $3 \%$ et fixé avec du glutaraldéhyde puis du tétroxyde d'osmium (Hayat, 1981) en présence de rouge de ruthénium (Luft, 1971). La déshydratation était réalisée par l'acétone et l'inclusion en Epon ainsi que la polymérisation de la résine se déroulaient à $60^{\circ} \mathrm{C}$ pendant $48 \mathrm{~h}$. Des coupes ultra-fines (microtome Ultracut Reichert, Vienne, Autriche) étaient déposées sur des grilles recouvertes d'un film de collodion, contrastées à l'acétate d'uranyle ( $4 \%$ en solution aqueuse) et au citrate de plomb (Venable et Coggeshall, 1965). Des coupes ultra-fines étaient également colorées selon la technique de Thiéry (1967) pour détecter la présence de polysaccharides. Les observations étaient réalisées avec un appareil Zeiss EM 10 (Oberkochen, RFA).

La méthode de Bradford (1976) était utilisée pour évaluer la quantité de protéines libérées au cours de la privation nutritionnelle par les bactéries dans la solution tampon et celle des protéines intracellulaires après hydrolyse alcaline des cellules par $\mathrm{NaOH} 3 \mathrm{~N}$ à $100^{\circ} \mathrm{C}$ pendant 20 min. L'albumine sérique bovine (Sigma) était employée comme standard.

Les acides aminés libres intracellulaires étaient dosés après chauffage des cellules dans l'eau distillée à $100^{\circ} \mathrm{C}$ pendant $20 \mathrm{~min}$; les acides aminés constitutifs étaient estimés après hydrolyse acide par $\mathrm{HCl} 6 \mathrm{~N}$ à $110^{\circ} \mathrm{C}$ pendant $24 \mathrm{~h}$. La détermination quantitative de chacun des acides aminés était obtenue à l'aide d'un analyseur automatique (Biotronik LC 5000, Münich, RFA).

L'ADN était extrait 3 fois avec $\mathrm{HClO}_{4}$ 0,5 $\mathrm{M}$ a $70^{\circ} \mathrm{C}$ pendant $15 \mathrm{~min}$ (Herbert et al, 1971) puis testé à l'aide de la diphénylamine (Burton, 1956). Le culot cellulaire avait été préalablement extrait avec $\mathrm{HClO}_{4} 0,25 \mathrm{M}$ pendant $30 \mathrm{~min}$ à $4^{\circ} \mathrm{C}$ pour éliminer les acides solubles (Munro et Fleck, 1966). L'ADN de thymus de veau (Sigma) était utilisé comme standard.

L'ARN était extrait avec $\mathrm{HClO}_{4} 0,5 \mathrm{M}$ à $37^{\circ} \mathrm{C}$ pendant $2 \mathrm{~h}$ (Trevelyan et Harrison, 1956) et dosé à l'aide d'orcinol selon la méthode de Herbert et al (1971). Le culot cellulaire avait été préalablement extrait avec $\mathrm{HClO}_{4} 0,25 \mathrm{M}$ pendant 30 min à $4^{\circ} \mathrm{C}$ pour éliminer les acides solubles (Munro et Fleck, 1966). L'ARN de levure (Sigma) était utilisé comme standard.

L'ATP intracellulaire était dosé, après extraction avec du diméthylsulfoxide pur (Merck), par bioluminescence avec un système luciférineluciférase à l'aide d'un bioluminomètre (Biolumat LB 9500T, Berthold, Wildbad, RFA).

L'activité de la lactate déshydrogénase (LDH) était dosée dans le surnageant de la suspension bactérienne selon la technique de Thomas (1975). L'activité LDH était également évaluée après traitement du culot cellulaire à $37^{\circ} \mathrm{C}$ pendant $3 \mathrm{~h}$ par le lysozyme $\left(1 \mathrm{mg} \cdot \mathrm{ml}^{-1}\right)$ en présence de $\mathrm{MgCl}_{2}, 6 \mathrm{H}_{2} \mathrm{O}, 20 \mathrm{mM}$.

\section{Reproductibilité}

Dans chaque cas, nous avons vérifié que les constituants dosés étaient stables dans les conditions de stockage choisies. Les résultats présentés ici sont la moyenne d'au moins 3 essais indépendants. Les variations observées étaient toujours inférieures à $10 \%$.

\section{RÉSULTATS}

\section{Viabilité}

L'utilisation d'un broyeur (Ultra-Turrax) préconisée par Martley (1972) pour réduire la 
longueur des chaînes bactériennes à la même taille moyenne n'est pas possible, car elle entraîne en soi une perte de viabilité (en moyenne, pour les 2 souches, après $20 \mathrm{~h}$ de privation nutritionnelle, moins de $0,01 \%$ de la valeur initiale, contre $85 \%$ sans emploi du broyeur).

La viabilité des cellules de Lc lactis représente $50 \%$ de sa valeur initiale après $21 \mathrm{~h}$ pour la souche CNRZ 125 ou $30 \mathrm{~h}$ pour la souche CNRZ 141 (fig 1). Elle devient négligeable beaucoup plus tard chez la souche CNRZ 125 (110 h) que chez la souche CNRZ 141 (50 h).

Les cellules de Lc lactis CNRZ 125 et CNRZ 141 transférées en tampon phosphate sont plus sensibles à la privation nutritionnelle lorsque leur croissance en milieu M 17 est arrêtée au cours de la phase exponentielle (12 et $22 \%$ de viabilité, respectivement; tableau I) que lorsqu'elles sont prélevées au cours de la phase stationnaire de croissance ( 70 et $59 \%$, respectivement).

La concentration en lactose du milieu M 17 apparaît comme un facteur très discriminant entre les souches (tableau I). Lorsqu'elle augmente de 5 à $50 \mathrm{~g}^{\circ} \mathrm{l}^{-1}$, la viabilité des cellules de Lc lactis CNRZ 125 fait plus que doubler (25 à 55\%) alors que celle des cellules de la souche CNRZ 141 devient 4 fois moins importante (50 à $12 \%)$. Si le lactose $\left(10{\mathrm{~g} \cdot \mathrm{I}^{-1}}^{-1}\right)$ est remplacé par le galactose $\left(5 \mathrm{~g}^{\circ} \mathrm{l}^{-1}\right)$, la viabilité des deux souches augmente alors qu'elle diminue si le glucose $\left(5 \mathrm{~g}^{-1}\right)$ remplace le lactose.

Quel que soit le stade de croissance auquel sont prélevées les cellules de $L c$ lactis CNRZ 125 et CNRZ 141 ou le milieu de culture dont elles proviennent, la densité optique de la suspension reste élevée et varie de façon moins marquée que la viabilité bactérienne (tableau I, fig 1). Toutefois, comme la viabilité, la $\mathrm{DO}_{650}$ est plus faible pour les cellules prélevées au cours de la phase exponentielle que pour les cellules prélevées plus tardivement.

\section{Morphologie}

Les chaînes bactériennes de Lc lactis CNRZ 125 sont initialement plus longues (5,2 cocci) que celles de la souche CNRZ 141 (tableau II). Après $30 \mathrm{~h}$ de séjour en tampon phosphate, le nombre de cocci par chaîne diminue, tout en restant supérieur à celui de la souche CNRZ 141, moins sensible aux conditions de culture préalable ou de privation nutritionnelle. La viabilité bactérienne, corrigée en tenant compte de la longueur moyenne des chaînes, est évi-

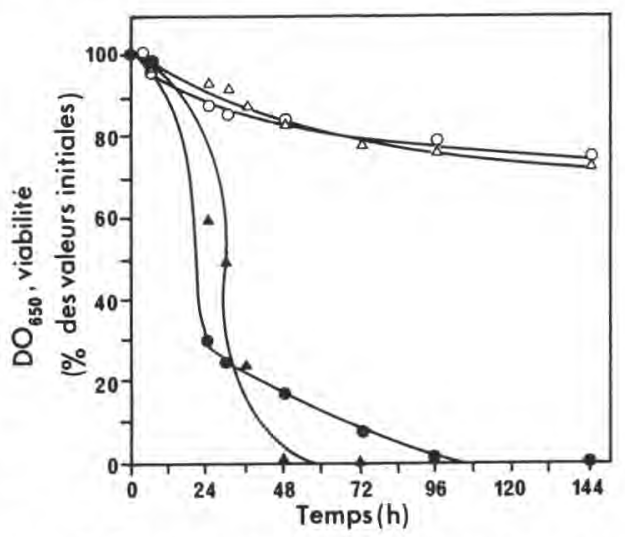

Fig 1. Évolution de la turbidité (symboles ouverts) de la suspension cellulaire et de la viabilité (symboles fermés) de Lactococcus lactis CNRZ 125 (cercle) et CNRZ 141 (triangle). Les cultures étaient arrêtées au cours de la phase stationnaire tardive de croissance en milieu M 17 et les cellules transférées en solution tampon phosphate de potassium $50 \mathrm{mM} \mathrm{pH} 6$.

Turbidity (open symbols) and viability (solid symbols) of Lactococcus lactis CNRZ 125 cells (circle) and CNRZ 141 (triangle) cell suspensions. Late stationary growth phase cells were harvested from $M 17$ medium and suspended in $50 \mathrm{mM}$ potassium phosphate buffer $\mathrm{pH} 6$. 
Tableau I. Variation de la $\mathrm{DO}_{650}$ des suspensions de cellules et de la viabilité (\% de la valeur initiale) dans une suspension de Lactococcus lactis CNRZ 125 et CNRZ 141 après $30 \mathrm{~h}$ de privation nutritionnelle en tampon phosphate $50 \mathrm{mM} \mathrm{pH} 6$. Les cellules étaient prélevées après 3 ou $6 \mathrm{~h}$ de croissance (phase exponentielle), et 12 ou $18 \mathrm{~h}$ (phase stationnaire).

Variation in $O D_{650}$ and viability (\% of initial value) in suspensions of Lactococcus lactis CNRZ 125 and CNRZ 141 cells after $30 \mathrm{~h}$ of starvation in $50 \mathrm{mM}$ potassium phosphate buffer $\mathrm{pH}$ 6. Cells were harvested after 3 or $6 \mathrm{~h}$ (exponential phase of growth) as well as 12 or $18 \mathrm{~h}$ (stationary phase) of incubation.

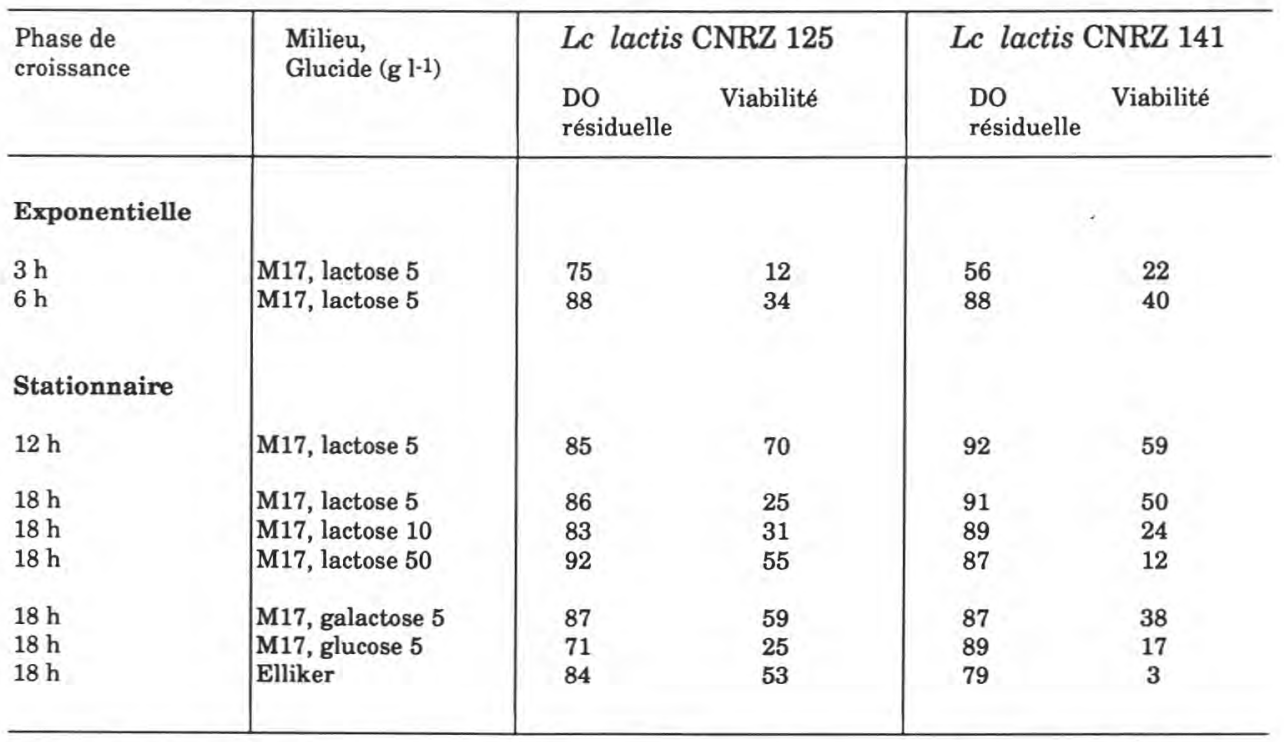

Tableau II. Évolution de la longueur moyenne des chaînes de Lactococcus lactis CNRZ 125 et CNRZ 141 au cours de la privation nutritionnelle. Les cellules, cultivées en milieu M 17 jusqu'en phase stationnaire $(18 \mathrm{~h})$, étaient transférées en tampon phosphate $50 \mathrm{mM} \mathrm{pH} 6$ pendant $30 \mathrm{~h}$ à $30^{\circ} \mathrm{C}$. La $\mathrm{DO}_{650}$ résiduelle et la viabilité sont exprimées en \% de leur valeur initiale.

Average chain length of Lactococcus lactis CNRZ 125 and CNRZ 141 during starvation. Stationary growth phase (18 h) cells were harvested from M17 medium and then transferred into $50 \mathrm{mM}$ phosphate potassium buffer $\mathrm{pH} 6$ for $30 \mathrm{~h}$ at $30^{\circ} \mathrm{C} . O D_{650}$ and cell viability are expressed as the \% of their initial value.

\begin{tabular}{l|cc|cc}
\hline & \multicolumn{2}{|c|}{ Lc lactis CNRZ 125 } & \multicolumn{2}{c}{ Lc lactis CNRZ 141 } \\
& $\begin{array}{l}\text { lactose } \\
10 \mathrm{~g} \mathrm{l}^{-1}\end{array}$ & $\begin{array}{c}\text { galactose } \\
5 \mathrm{~g} \mathrm{l}^{-1}\end{array}$ & $\begin{array}{c}\text { lactose } \\
10 \mathrm{~g} \mathrm{l}^{-1}\end{array}$ & $\begin{array}{c}\text { galactose } \\
5 \mathrm{~g} \mathrm{l}^{-1}\end{array}$ \\
\hline DO résiduelle & 83 & 87 & 89 & 97 \\
Cocci par chaîne : & 5,2 & 2,9 & 1,9 & 1,8 \\
$0 \mathrm{~h}$ & 3,0 & 2,2 & 1,7 & 1,6 \\
Viabilité & 31 & 59 & 24 & 38 \\
Viabilité corrigée & 18 & 45 & 21 & 34 \\
\hline
\end{tabular}


demment plus faible que la viabilité bactérienne déterminée par dénombrement des cellules sur milieu solide (tableau II).

Comme le montre l'examen en microscopie électronique, seule la souche LC lactis CNRZ 125 possède une couche périphérique extérieure à la paroi (slime) (fig $2 a, b)$ qui s'amincit au cours de l'incubation en solution saline tamponnée. Lors de la coloration de Thiéry, la réaction lente avec le thiocarbohydrazide et une coloration peu dense sous forme de grains épars, sont en faveur de la nature glycoprotéique et non polysaccharidique de cette structure.

Au cours de leur séjour en tampon, les cellules de Lc lactis CNRZ 125 et CNRZ 141 montrent dès $24 \mathrm{~h}$ une altération structurale importante et similaire. Une dégradation importante des ribosomes survient ainsi qu'une condensation du matériel cytoplasmique vers la périphérie des cellules (fig 2c) et une agrégation de I'ADN. Ces modifications concernent alors environ $70 \%$ des cellules de chaque souche tandis que des cellules normales persistent (tableau III).

Après $48 \mathrm{~h}$, les cellules de la souche CNRZ 125 ne montrent pas de lyse contrairement à la souche CNRZ 141 pour laquelle on note $20 \%$ de cellules lysées, les autres cellules étant altérées (tableau III). Cette moindre sensibilité des cellules de la souche CNRZ 125 au séjour en tampon phosphate, se retrouve après $120 \mathrm{~h}$. Les cellules lysées (fig 2d) sont en proportion nettement moindre chez la souche CNRZ 125 (5\% de cellules lysées) que chez la souche CNRZ 141 (34\%).

\section{Changements intracellulaires}

Après $28 \mathrm{~h}$ de séjour en tampon phosphate, la dégradation des protéines est plus faible chez la souche CNRZ 125 (respectivement 36 et $32 \%$ de perte pour les cellules cultivées en milieu M 17 ou Elliker) que chez la souche CNRZ 141 ( $50 \%$ quel que soit le milieu) (tableau IV).

Tableau III. Observation en microscopie électronique de la morphologie des cellules de Lactococcus lactis CNRZ 125 et CNRZ 141 au cours de leur séjour en tampon phosphate $50 \mathrm{mM} \mathrm{pH} \mathrm{6.} \mathrm{Les} \mathrm{cel-}$ lules étaient cultivées en milieu $M 17$ (lactose $10 \mathrm{~g} \cdot \mathrm{I}^{-1}$ ) et prélevées au cours de la phase stationnaire de croissance ( $18 \mathrm{~h}$ ). Le nombre de cellules de chaque forme est exprimé en $\%$ du total des cellules. Electron microscopic observations of Lactococcus lactis CNRZ 125 and CNRZ 141 cells suspended in $50 \mathrm{mM}$ potassium phosphate buffer $\mathrm{pH}$ 6. Cells grown in $\mathrm{M} 17$ medium (lactose $10 \mathrm{~g}^{\circ \mathrm{F}^{-1}}$ ) were harvested during stationary phase $(18 \mathrm{~h})$. The number of each cell type is expressed as the \% of the total population.

\begin{tabular}{|c|c|c|c|c|c|c|}
\hline \multirow[b]{2}{*}{$\begin{array}{l}\text { temps } \\
\text { (h) }\end{array}$} & \multicolumn{3}{|c|}{ Lc lactis CNRZ 125} & \multicolumn{3}{|c|}{ Lc lactis CNRZ 141} \\
\hline & normales & $\begin{array}{l}\text { cellules } \\
\text { altérées }\end{array}$ & lysées & normales & $\begin{array}{l}\text { cellules } \\
\text { altérées }\end{array}$ & lysées \\
\hline 24 & 34 & 66 & 0 & 24 & 73 & 3 \\
\hline 48 & 5 & 95 & 0 & 0 & 80 & 20 \\
\hline 96 & 2 & 97 & 1 & 0 & 70 & 30 \\
\hline 120 & 0 & 95 & 5 & 0 & 66 & 34 \\
\hline
\end{tabular}



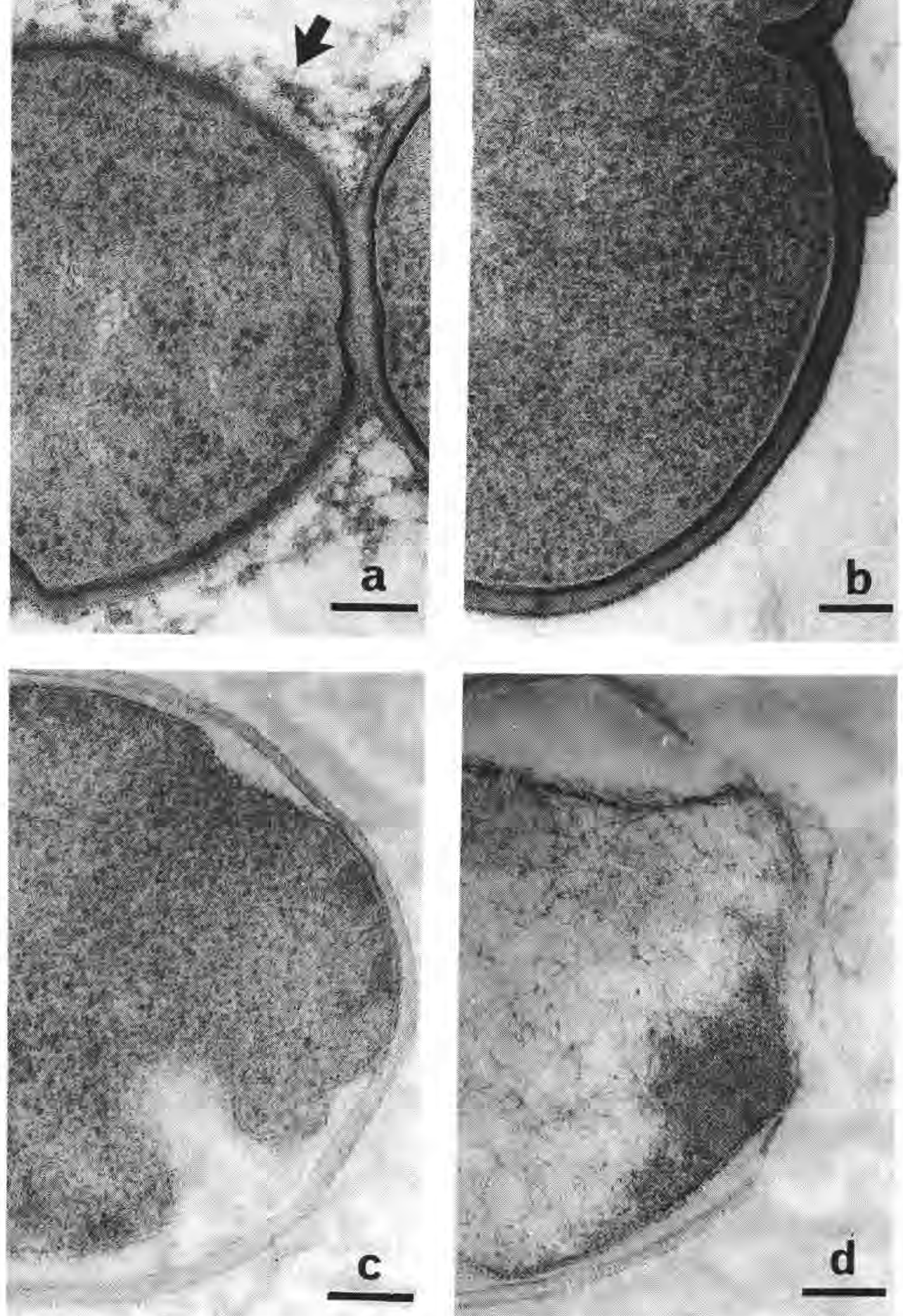

Fig 2. Morphologie des cellules de Lactococcus lactis en microscopie électronique (fixation en présence de rouge de ruthénium, coloration sur coupes en acétate d'uranyle et citrate de plomb). Les cellules étaient prélevées au cours de la phase stationnaire de croissance (18 h) en milieu $\mathrm{M} 17$ (lactose $10 \mathrm{~g} \cdot \mathrm{r}^{-1}$ ) puis transtérées en solution tampon phosphate $50 \mathrm{mM} \mathrm{pH} \mathrm{6.} \mathrm{Cellules} \mathrm{de} \mathrm{la} \mathrm{souche} \mathrm{CNRZ} \mathrm{125(a)} \mathrm{et} \mathrm{CNRZ} \mathrm{141(b)} \mathrm{avant} \mathrm{transfert} \mathrm{en} \mathrm{solution} \mathrm{tampon,} \mathrm{la}$ couche périphérique extérieure à la paroi est indiquée par une flèche (a). Cellule altérée (c) ou en cours de lyse (d) de la souche CNRZ125 après $120 \mathrm{~h}$ en solution tampon. Barre $=100 \mathrm{~nm}$.

Electron microscopic observation of Lactococcus lactis cell morphology (fixed in the presence of ruthenium red, stained with uranyl acetate and lead citrate). Late stationary growth phase (18 h) cells were harvested from $M 17 \mathrm{me}$ dium (lactose $10 \mathrm{~g}^{\circ} \mathrm{H}^{-1}$ ) and transferred into $50 \mathrm{mM}$ phosphate buffer $\mathrm{pH}$. Cells of strain CNRZ 125 (a) and CNRZ 141 (b) before transferring into buffer, arrow designates slime formation (a). Altered (c) or during lysing (d) cells of strain CNRZ 125 after $120 \mathrm{~h}$ of incubation in buffer. Bar $=100 \mathrm{~nm}$. 
Tableau IV. Évolution des concentrations de protéines, acides aminés libres, ADN et ARN intracellulaires de Lactotoccus lactis CNRZ 125 et CNRZ 141 au cours de la privation nutritionnelle. Les cellules étaient cultivées en milieu M 17 (lactose $10 \mathrm{~g}^{-1}{ }^{-1}$ ) ou Elliker jusqu'en phase stationnaire (18 h) et transférées en tampon phosphate de potassium $50 \mathrm{mM} \mathrm{pH}$ 6. Les résultats sont exprimés en $\mathrm{mg}$ par g de poids sec initial, les pourcentages de perte correspondants sont indiqués entre parenthèses.

Decrease in protein, free amino acid, intracellular DNA and RNA concentrations of Lactococcus lactis CNRZ 125 and CNRZ 141 during starvation. Stationary growth phase (18 h) cells were harvested

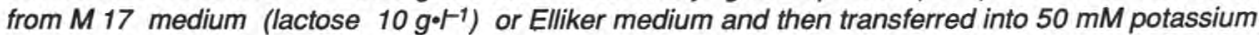
phosphate $\mathrm{pH}$. Results are expressed in $\mathrm{mg}$ per $\mathrm{g}$ of initial dry weight; the \% of loss was calculated and is indicated in parentheses.

\begin{tabular}{l|l|cc|cc}
\hline Souche & \multicolumn{2}{|l|}{$\begin{array}{l}\text { Durée de la } \\
\text { privation } \\
\text { nutritionnelle }\end{array}$} & \multicolumn{2}{|c|}{ Protéines } & \multicolumn{2}{c}{ Acides aminés } \\
& & E17 & Elliker & M17 & Elliker \\
\hline CNRZ & $0 \mathrm{~h}$ & 458 & 452 & 21 & 26 \\
125 & $28 \mathrm{~h}$ & 283 & 306 & 16 & 22 \\
& & $(36)$ & $(32)$ & $(24)$ & $(15)$ \\
& & & & & \\
\hline CNRZ & $0 \mathrm{~h}$ & 449 & 450 & 24 & 29 \\
141 & $28 \mathrm{~h}$ & 223 & 226 & 18 & 19 \\
& & $(50)$ & $(50)$ & $(25)$ & $(34)$ \\
\hline
\end{tabular}

\begin{tabular}{|c|c|c|c|c|c|}
\hline \multirow[t]{2}{*}{ Souche } & \multirow{2}{*}{$\begin{array}{l}\text { Durée de la } \\
\text { privation } \\
\text { nutritionnelle }\end{array}$} & \multicolumn{2}{|c|}{$\mathrm{ADN}$} & \multicolumn{2}{|c|}{ ARN } \\
\hline & & M17 & Elliker & M17 & Elliker \\
\hline \multirow[t]{2}{*}{$\begin{array}{l}\text { CNRZ } \\
125\end{array}$} & $\begin{array}{c}0 \mathrm{~h} \\
28 \mathrm{~h}\end{array}$ & $\begin{array}{l}31 \\
28\end{array}$ & $\begin{array}{l}21 \\
20\end{array}$ & $\begin{array}{l}215 \\
135\end{array}$ & $\begin{array}{l}221 \\
161\end{array}$ \\
\hline & & (10) & (5) & (37) & (27) \\
\hline \multirow[t]{2}{*}{$\begin{array}{l}\text { CNRZ } \\
141\end{array}$} & $\begin{array}{c}0 \mathrm{~h} \\
28 \mathrm{~h}\end{array}$ & $\begin{array}{l}29 \\
28\end{array}$ & $\begin{array}{l}28 \\
26\end{array}$ & $\begin{array}{l}218 \\
179\end{array}$ & $\begin{array}{l}221 \\
176\end{array}$ \\
\hline & & (3) & (7) & (18) & (20) \\
\hline
\end{tabular}

Les acides aminés constitutifs représentent $52 \%$ du poids sec bactérien initial. Les acides glutamique et aspartique ainsi que l'alanine sont les principaux acides aminés constitutifs. Lorsque les cultures sont réalisées en milieu Elliker, la dégradation des acides aminés libres est environ deux fois moins importante chez la souche CNRZ 125 que chez la souche CNRZ 141 (respectivement 15 et $34 \%$ de perte; tableau IV). Par contre, elle est identique chez la souche CNRZ 125 et CNRZ 141 lorsque les cellules sont cultivées en milieu M 17 (environ 25\%).

La quantité d'ADN intracellulaire diminue peu. Après $28 \mathrm{~h}$, elle représente entre 90 et $97 \%$ de la valeur initiale pour les souches CNRZ 125 et CNRZ 141 (tableau IV).

Chez les cellules de la souche CNRZ 125 , l'ARN ne représente plus que 63 ou $73 \%$ de sa valeur initiale selon que la cul- 
ture est réalisée en milieu M 17 ou en milieu Elliker tandis que pour la souche CNRZ 141, environ $81 \%$ de l'ARN intracellulaire subsiste quel que soit le milieu de culture dont proviennent les cellules (tableau IV).

La quantité intracellulaire d'ATP des souches CNRZ 125 et CNRZ 141 cultivées en milieu M 17 additionné de lactose (10 $\mathrm{g}^{\circ} \mathrm{l}^{-1}$ ) diffère initialement (respectivement 0,26 et $0,48 \mathrm{mg}$ par $\mathrm{g}$ de poids sec initial). Au cours du séjour en tampon, la diminution d'ATP suit la même cinétique pour les deux souches, soit une diminution de $50 \%$ en $3 \mathrm{~h}$ et de $93 \%$ en $8 \mathrm{~h}$. La quantité intracellulaire d'ATP représente alors pour chaque souche moins de $1 \%$ de la valeur maximale mesurée au cours de la croissance.

\section{Libération de composés solubles}

Au cours de leur séjour en tampon phosphate, les cellules de la souche CNRZ 125 libèrent plus de protéines (2 ou $5 \mathrm{mg}$ par $\mathrm{g}$ de poids sec initial selon que la culture est réalisée en milieu Elliker ou $M$ 17) que la souche CNRZ 141 (1 mg par g de poids sec initial, quel que soit le milieu de culture). Par contre, la perte des acides aminés est moins importante chez la souche CNRZ 125 (19 mg par g de poids sec initial quel que soit le milieu de culture) que chez la souche CNRZ 141 (24 ou 27 mg par $\mathrm{g}$ de poids sec initial selon que la culture est réalisée en milieu Elliker ou M 17).

Dans tous les cas, après $28 \mathrm{~h}$ de privation nutritionnelle, la quantité d'acides aminés en solution dans le tampon phosphate est supérieure à la quantité d'acides aminés libres intracellulaires perdus. Cette différence provient vraisemblablement de la libération des produits d'hydrolyse des protéines qui s'ajoutent aux acides aminés intracellulaires libérés en solution.
Au cours de leur séjour en tampon phosphate, les cellules de Lc lactis CNRZ 125 libèrent peu ( $18 \mu$ Kat par $g$ de poids sec initial) ou pas de LDH selon que leur croissance est réalisée en milieu $M 17$ additionné de galactose $\left(5 \mathrm{~g}^{-1}\right)$ ou de lac-

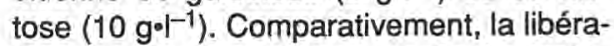
tion de LDH par les cellules de Lc lactis CNRZ 141 est beaucoup plus importante $(1,87$ et 1,97 mKat par $\mathrm{g}$ de poids sec initial, respectivement) dans les mêmes conditions. Ceci représente $2 \%$ de l'activité enzymatique intracellullaire pour Lc lactis CNRZ 125 et $7,5 \%$ pour la souche CNRZ 141.

\section{DISCUSSION}

L'étude de la physiologie de Lc lactis CNRZ 125 et CNRZ 141 en conditions de privation nutritionnelle met en évidence une évolution différente pour la plupart des facteurs étudiés.

La souche CNRZ 125 se démarque bien de la souche CNRZ 141 par une perte de viabilité plus faible, des changements morphologiques, une dégradation des protéines et des acides aminés ainsi qu'une libération d'acides aminés et d'activité LDH moins importante que chez la souche CNRZ 141. Par ailleurs, les chaînes bactériennes de CNRZ 125 sont plus longues, ce qui témoignerait d'une activité autolytique plus faible (Mc Donald, 1971).

Seules les cellules de la souche CNRZ 125 possèdent une couche périphérique extérieure à la paroi dont la structure serait intéressante à connaître, la coloration de Thiéry suggérant une nature glycoprotéique. Seuls, Macura et Townsley (1984) avaient décrit une telle couche glycoprotéique dans le cas de la sous-espèce cremoris de Lc lactis alors que de nombreux travaux indiquent la présence de protéines (Sundman, 1953; Nilsson et Nilsson, 1958; 
Rimpiläinen et al, 1986; Kontusaari et Forsén, 1987, 1988) pour les sousespèces cremoris ou lactis.

La couche externe mise en évidence chez CNRZ 125 pourrait être un caractère déterminant pour expliquer sa moindre activité autolytique par rapport à la souche CNRZ 141. Elle peut représenter pour la cellule une barrière de protection contre l'environnement extérieur (Rogers et al, 1980) ou modifier l'activité de la ou des enzyme(s) autolytique(s) à l'image de ce qui a été proposé pour l'acide téichoïque associé à la membrane et les polymères liés au peptidoglycane par Niskasaari (1989).

Une partie de nos résultats confirment pour la souche CNRZ 125 de la variété diacetylactis de Lc lactis des données importantes relatives à la physiologie de $L C$ lactis en conditions de privation nutritionnelle. C'est le cas, en particulier, de la viabilité qui est d'autant plus faible que le métabolisme endogène, qui concerne essentiellement les protéines et l'ARN, est plus rapide. Ce phénomène a déjà été mis en évidence pour Lc lactis ML3 (Thomas et Batt, 1968) ou pour d'autres espèces bactériennes (Ensign, 1970; Dawes, 1976; Boylen et Mulks, 1978). De même, la perte de viabilité ne peut pas être associée à celle d'un constituant ou d'une fonction cellulaire spécifique (Thomas et Batt, 1968; Dawes, 1976).

Certaines données de cette étude relatives à la souche CNRZ 141 (ML3) diffèrent parfois sensiblement de celles de Thomas et Batt (1968). Ces auteurs avaient utilisé des conditions d'expérience (cellules prélevées au cours de la phase exponentielle de croissance, tampon phosphate de potassium et de sodium, addition éventuelle d'EDTA ou de $\mathrm{Mg}^{2+}$ ) différentes des nôtres.

La composition des cellules des souches de Lc lactis CNRZ 125 et CNRZ 141 est sensible aux conditions de culture.
Cette sensibilité pourrait s'expliquer par une utilisation différentielle des composés comme précurseurs des macromolécules constitutives des cellules ou par un environnement plus ou moins favorable au métabolisme des précurseurs.

La perte de viabilité à la fois de Lc Lactis CNRZ 125 et CNRZ 141 est due, en partie seulement, à la lyse modérée observée en microscopie électronique et qui correspond à la diminution de $\mathrm{DO}_{650}$ et pour le reste à des modifications (structurales, biochimiques, énergétiques) fatales aux cellules. La lyse plus importante des cellules prélevées au cours de la phase exponentielle de croissance s'expliquerait par l'activité accrue des autolysines au cours de cette phase (Young, 1966) comme cela a déjà été suggéré dans le cas de Lc lactis subsp cremoris (Mou et al, 1976; Niskasaari, 1989).

L'effet négatif du glucose sur la viabilité bactérienne des 2 souches étudiées pourrait s'expliquer par l'incapacité des cellules à utiliser cet ose comme précurseur adéquat de la galactosamine présente dans la paroi bactérienne (Moustafa et Collins, 1968). Par ailleurs, il serait intéressant de tester plus amplement la relation entre la concentration de lactose et la lyse et d'expliquer le phénomène. La souche CNRZ 125 devrait voir sa viabilité chuter notablement dans les fromages où le lactose est rapidement et complètement utilisé.

La souche Lc lactis CNRZ 125 montre des particularités intéressantes au niveau de sa physiologie qui pourraient constituer effectivement un critère de sélection supplémentaire des levains lactiques comme cela a déjà été proposé par Vegarud et al (1983) et Niskasaari (1989) en plus des critères habituels, notamment la production d'acide ou celle d'arôme. II serait notamment intéressant de savoir si l'on a intérêt à utiliser, en fromagerie, des souches à lyse rapide ou non, dans la mesure où 
les cellules non lysées peuvent intervenir éventuellement comme réservoir d'activités enzymatiques.

\section{REMERCIEMENTS}

Nous remercions $D$ Le Bars pour le dosage des acides aminés et $\mathrm{C}$ Le Gallo pour sa collaboration technique en microscopie électronique.

\section{RÉFÉRENCES}

Boylen CW et Mulks MH (1978) The survival of coryneform bacteria during periods of prolonged nutrient starvation. $J$ Gen Microbiol 105, 323-334

Bradford MM (1976) A rapid and sensitive method for the quantitation of microgram quantities of protein utilizing the principle of proteindye binding. Anal Biochem 72, 248-254

Burton K (1956) A study of the conditions and mechanism of the diphenylamine reaction for the colorimetric estimation of deoxyribonucleic acid. Biochem J 62, 315-322

Choisy C, Desmazeaud M, Gripon JC, Lamberet G, Lenoir J, Tourneur C (1987) Les phénomènes microbiologiques et enzymatiques et la biochimie de l'affinage. In: Le fromage (A Eck, ed). Lavoisier, Paris, 62-100

Dawes EA (1976) Endogenous metabolism and the survival of starved prokaryotes. In: The survival of vegetative microbes. (TRG Gray and JR Postgate, eds). Cambridge University Press, 19-53

Ensign JC (1970) Long-term starvation survival of rod and spherical cells of Arthrobacter crystallopoietes. J Bacteriol 103, 569-577

Foucaud C, Furlan S, Winters DA, Hemme D (1990) Specific loss of the plasmid encoding for lactose metabolism by Lactococcus lactis. Milchwissenschaft 45, 642-646

Hayat HA (1981) Fixation for electron microscopy. (Van Nostrand, Rheinhold Co, eds) Academic Press, London, New York, 77-87

Herbert D, Phipps PJ, Strange RE (1971) Chemical analysis of microbial cells. In: Methods in microbiology. Vol 5B (JR Norris, DW Ribbons, eds) Academic Press, London, 210344
Kontusaari S, Forsén R (1987) Cell surface proteins of encapsulated Streptococcus cremoris: identification and immunochemical characterization. J Appl Bacteriol 63, 133-137

Kontusaari S, Forsén R (1988) Finnish fermented milk "Viili": involvement of two cell surface proteins in production of slime by Streptococcus lactis ssp cremoris. J Dairy Sci 71, 31973202

Langsrud T, Laandas A, Castberg HB (1987) Autolytic properties of different strains of group N streptococci. Milchwissenschaft 42, 556-560

Lenoir J, Lamberet G, Schmidt JL, Tourneur C (1985) La maîtrise du bioréacteur fromage. Biofutur 41, 23-49

Luft JH (1971) Ruthenium red and violet. 1. Chemistry, purification, methods of use for electron microscopy and mechanism of action. Anat Rec 171, 347-368

McDonald IJ (1971) Filamentous forms of Streptococcus cremoris and Streptococcus lactis. Observations on structure and susceptibility to lysis. Can J Microbiol 17, 897-902

Macura D, Townsley PM (1984) Scandinavian ropy milk. Identification and characterization of endogenous ropy lactic streptococci and their extracellular excretion. J Dairy Sci 67 , 735-744

Martley FG (1972) The effect of cell numbers in streptococcal chains on plate-counting. $N Z J$ Dairy Sci Technol 7, 7-11

Mou L, Sullivan JJ, Jago GR (1976) Autolysis of Streptococcus cremoris. J Dairy Res 43, 275282

Moustafa HH, Collins EB (1968) Role of galactose or glucose-1-phosphate in preventing the lysis of Streptococcus diacetylactis. J Bacteriol 95, 592-602

Munro HN, Fleck A (1966) The determination of nucleic acids. In: Methods of biochemical analysis. Vol 14 (D Glick, ed). Interscience Publishers, New York, 113-176

Nilsson R, Nilsson G (1958) Studies concerning Swedish ropy milk. The antibiotic qualities of ropy milk. Arch Microbiol 31, 191-197

Niskasaari K (1989) Characteristics of the autolysis of variants of Lactococcus lactis subsp cremoris. J Dairy Res 56, 639-649

Rimpiläinen MA, Niskasaari $\mathrm{K}$, Juutinen KMS, Nurmiaho-Lassila EL, Forsén R (1986)The 
plasma membrane of Streptococcus cremoris: isolation and partial characterization. $J$ Appl Bacteriol 60, 389-394

Rogers HJ, Perkins HR, Ward JB (1980) Microbial cell walls and membranes. Chapman and Hall, London

Sundman V (1953) On the protein character of a slime produced by Streptococcus cremoris in Finnish ropy sour milk. Acta Chem Scand 7, 558-560

Terzaghi BE, Sandine WE (1975) Improved medium for lactic streptococci and their bacteriophages. Appl Microbiol 29, 807-813

Thiéry JP (1967) Mise en évidence des polysaccharides sur coupes fines en microscopie électronique. J Microsc 6, 987-1018

Thomas TD (1975) Tagatose-1,6-diphosphate activation of lactate deshydrogenase from Streptococcus cremoris. Biochem Biophys Res Commun 63, 1035-1042

Thomas TD, Batt RD (1968) Survival of Streptococcus lactis in starvation conditions. J Gen Microbiol 50, 367-382
Thomas TD, Batt RD (1969) Degradation of cell constituents by starved Streptococcus lactis in relation to survival. J Gen Microbiol 58, 347-362

Thomas TD, Lyttleton P, Williamson KI, Batt RD (1969) Changes in permeability and ultrastucture of starved Streptococcus lactis in relation to survival. J Gen Microbiol 58, 381390

Trevelyan WE, Harrison JS (1956) Studies on yeast metabolism. 7. Yeast carbohydrate fractions. Separation from nucleic acid, analysis, and behaviour during anaerobic fermentation. Biochemistry 63, 23-33

Vegarud G, Castberg HB, Langsrud T (1983) Autolysis of group $\mathrm{N}$ streptococci. Effects of media composition and temperature. $J$ Dairy Sci 66, 2294-2302

Venable JH, Coggeshall R (1965) A simplified lead citrate stain for use in electron microscopy. J Cell Biol 25, 407-408

Young FE (1966) Autolytic enzyme associated with cell walls of Bacillus subtilis. J Biol Chem 241, 3462-3467 